\title{
A Vaccine Is Coming but Prevention Is Still up to Us
}

\author{
Hector Ortega \\ Gossamer Bio, Inc., Clinical Development, San Diego, CA, USA \\ Email: hortega@gossamerbio.com
}

How to cite this paper: Ortega, H. (2021) A Vaccine Is Coming but Prevention Is Still up to Us. Open Journal of Nursing, 11, 1-6. https://doi.org/10.4236/ojn.2021.111001

Received: November 26, 2020

Accepted: January 11, 2021

Published: January 14, 2021

Copyright (c) 2021 by author(s) and Scientific Research Publishing Inc. This work is licensed under the Creative Commons Attribution International License (CC BY 4.0).

http://creativecommons.org/licenses/by/4.0/

\begin{abstract}
Despite having a better understanding of the COVID-19 disease, we are facing significant challenges regarding behaviors that enforce the lack of adherence to public health recommendations including the use of face masks and vaccine acceptability. Lessons learned from the 1918 flu pandemic, highlight the importance of wearing a face mask in conjunction with adherence to other public health recommendations. There are two key factors that influence how our communities are translating scientific evidence, denialism and ignorance. Sadly, a real consequence of this pandemic among a significant number of patients with COVID-19 disease is denial. As health care providers we need to take part in addressing these issues and educate our communities especially during this time where we see a second and larger uptake of COVID-19 cases. Fighting denialism and ignorance represents a significant challenge to our society and could have a substantial positive impact on helping with vaccine acceptability and other public health recommendations.
\end{abstract}

\section{Keywords}

COVID-19, Education, Denialism, Ignorance, Pandemic, SARS-CoV-2

\section{Introduction}

Since the start of the COVID-19 pandemic in December 2019, there have been more than 250,000 deaths in the US alone. That is more people than strokes, suicides and car crashes kill combined in a full year. To put this in perspective it is important to go back in time and look at the lessons learned from prior experience. A good example is the 1918 H1N1 pandemic also known as the "Spanish flu" that killed about 50 million people worldwide, including an estimated 675,000 people in the US [1].

It is well established that respiratory infections are transmitted through infec- 
tious particles small enough to be suspended in air. Specifically, respiratory infections occur through transmission of virus-containing droplets ( $>5$ to $10 \mu \mathrm{m}$ ) and aerosols $(\leq 5 \mu \mathrm{m})$ exhaled from infected individuals during breathing, speaking, coughing, and sneezing [2]. A large proportion of the spread of COVID-19 appears to be occurring through airborne transmission by asymptomatic individuals simply by breathing and speaking. Aerosols can accumulate, remain infectious in indoor air for hours, and are easily inhaled. There is evidence that respiratory, corneal, and intestinal epithelial cells are selectively a rich source for the expression of genes (ACE2 and TMPRSS2) linked to the cellular entry of coronaviruses that trigger innate immunity, highlighting their potential role in initial viral infection, spread, and clearance [3]. Notably, nasal epithelial cells have the highest expression among all investigated cells in the respiratory tract. SARS-CoV-2 has been shown to replicate three times faster than SARS-CoV-1 and thus can rapidly spread to the pharynx from which it can be shed before the innate immune response becomes activated and produces symptoms. Thus, the use of preventive measures, particularly face masks, remains a critical part to control the spread of the disease.

\section{The Past}

In 1918, the world was still engaged in World War I. Movement and mobilization of troops placed large numbers of people in close contact and living spaces were overcrowded. Health services were limited, and up to $30 \%$ of US physicians were deployed to military service [1]. In addition, medical technology and countermeasures at the time were limited or non-existent. No diagnostic test existed that could detect influenza infection. In fact, doctors didn't know influenza viruses existed. Many health experts at the time thought the 1918 pandemic was caused by a bacterium called "Pfeiffer's bacillus," known now as Haemophilus influenzae [4]. No antiviral drugs were available. Critical care measures, such as intensive care support and mechanical ventilation also were not readily available. Without these treatment capabilities, doctors were left with few options other than supportive care. Some cities in the US managed to implement community mitigation measures, such as closing schools, banning public gatherings, and issuing isolation or quarantine orders, but the federal government had no centralized role in helping to plan or initiate these interventions [1] [4]. In fact, some church leaders hosted open air services, as they thought short meetings in sufficiently ventilated churches would not seriously harm communities. However, recognizing the danger, health authorities and law enforcement intervened in some places, either by discouraging the services, denying permission to have them, or dispatching officers to meetings. At the height of the full wave, some pastors and rabbis used their buildings as makeshift hospitals. An analysis of the 1918 pandemic noted that cities without early implementation of strict prevention measures fared more poorly than cities where some degree of intervention occurred early on [4] [5]. The effectiveness of the face masks used during that pandemic was perceived to be low, but this was attributed to the poor quality of 
mask material and inappropriate use of the masks, likely propelled by some level of ignorance.

\section{The Present}

Back in the early months of 2020 the effectiveness of face mask use in community settings for the prevention of COVID-19 infection or transmission met controversy and confusion. Current strategies for infection control practices, such as avoiding poorly ventilated places, staying at home if you are sick, and avoiding people who are coughing are not vastly different than what was recommended a century ago. In fact, during the 1918 pandemic, the very same control measures were utilized: face masks, limiting crowds, personal hygiene training, isolation and quarantine of cases, and sputum control measures [1]. Strategy is useful for planning purposes and for determining appropriate mitigations based on the severity of a pandemic as long as it is properly implemented and in a timely manner. Nurses and clinicians as well as public health experts should engage in dialogue on issues of public health such as proper mask use, vaccine acceptability, and the appropriate use of medications.

Countries that have been most effective in reducing the spread of COVID-19 in the early months of the pandemic implemented universal face mask use from the outset of the pandemic, as demonstrated in mainly Asian countries. In some countries the pattern of cases from onset to near control reflected a bell-shaped curve, while in others, the curve was skewed to the right (Figure 1), possibly due to the extended rate of transmission and limited early implementation of mitigation strategies. Notably, some Western countries with higher rates were slow to implement the use of face covering more broadly, including the US. Modelling studies suggest that even a small reduction in community transmission has the potential to make a major impact on health care system burden, for example hospital bed space and ventilators.

\section{The Challenge}

While COVID-19 is predominantly self-limiting, up to $20 \%$ of symptomatic individuals will progress to severe or critical disease with clinical manifestations including pneumonia, acute respiratory distress syndrome, multiorgan system dysfunction, hypercoagulation, and hyperinflammatory manifestations [6]. Inevitably this is creating a significant stress in the health care system by competing for hospital bed and ICU availability. In addition, other chronic conditions requiring control such as hypertension and diabetes will be pushed to the back burner.

A growing body of data [7] [8] suggests that some patients with COVID-19, including individuals with mild symptoms, will develop a variably prolonged course of recovery, including fatigue, cognitive impairment, and cardiopulmonary dysfunction. These individuals are called the "long haulers": those who still have not fully recovered from COVID-19 weeks or even months after symptoms 

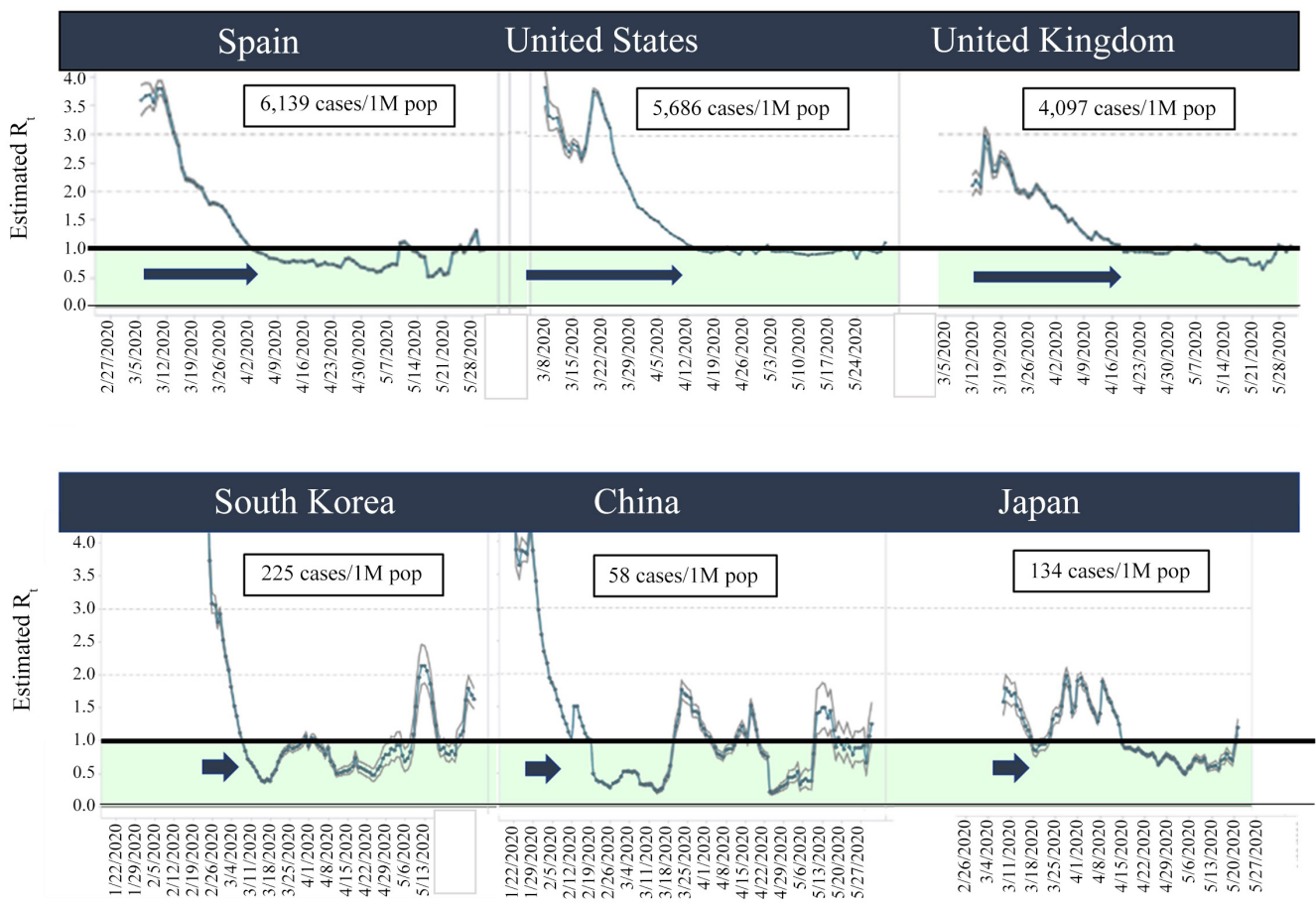

Figure 1. Contrast of effective reproduction number (Rt) estimates across selected Western and Asian countries during the early days of the COVID-19 pandemic. Number of cases per 1,000,000 population in selected countries as of June 2,2020. Rt refers to the average number of individuals infected by one individual and reflects interventions such as social distancing and wearing a face mask. The black horizontal line indicates $\mathrm{Rt}=1$, below which sustained transmission is unlikely so long as measures against transmission are maintained, indicating that the outbreak is under control. The arrows represent the duration between onset of cases until reaching a Rt of 1.0. Sources TIBCO, COVID-19 Live Report and Worldometer (June 2, 2020).

first presented. Approximately 10\% of people who have had COVID-19 will experience these prolonged symptoms [7]. This further highlights the importance for taking this disease seriously. While treatment options for patients with severe disease requiring hospitalization are now available, with corticosteroids emerging as the treatment of choice for critically ill patients, interventions that can be administered early during the course of infection to prevent disease progression and longer-term complications are urgently needed. Politicization of the vaccine and mask use has created widespread mistrust among the public. Thus, acceptability of the COVID-19 vaccines as well as recommendations for consistent use of a face mask in public will depend on proactive education in the community. While today considerable advancements have been made in health technology, disease surveillance, rapid access to information via the Internet, accessibility to ventilators, and new medicines including specific monoclonal antibody treatments and vaccines, we still are facing a significant death toll. Why does the death rate continue to climb? I believe this is occurring partly because of ignorance and denialism.

Merriam-Webster defines "ignorance" as the state or fact of being ignorant, i.e., lack of education, knowledge, or awareness. Some synonyms include clu- 
elessness, incognizance, nescience, obliviousness, unawareness, and unfamiliarity. On the other hand, denial, a rejection of scientific evidence, provides a fertile ground for ignorance. Denialism is an intensification of denial. Denialism rejects a proposition on which a scientific consensus exists [9]. While denial hides from the truth, denialism builds a new and "better" truth. This was exhibited earlier by anti-mask behavior and now by anti-vaccine beliefs. Despite all the available scientific evidence, still some people do not follow basic health recommendations. It clearly appears that anti-science rhetoric has made an impact and we are now facing the consequences with increased confirmed infections accompanied by ever increasing hospitalization rates and deaths. Currently, new outlets of social media are reinforcing false beliefs and discouraging people from seeking the truth. False beliefs about COVID-19 are also more likely to occur among people with limited capability for evaluating scientific information [10]. Communities may be able to prevent COVID-19 infections by wearing a mask, physical distancing, avoiding large gatherings, and in the very near future immune protection via vaccination. But it is more difficult today to protect people from misinformation because information is readily available at our fingertips. While only $4 \%$ of the world's population resides in the US, the US has accounted for about $20 \%$ of the world's deaths related to COVID-19 and has performed less well than several other developed nations despite having one of the most advance technological systems and research centers. Education matters, considering that ignorance is defined as a lack of education or knowledge. In a study that included 9654 US adults, $48 \%$ of those who had a high school education or less believed there was some truth to the conspiracy theory that COVID-19 was engineered as a bioweapon but only $15 \%$ among those with some postgraduate training endorsed this idea [10]. Notably, we have not seen an improvement in our educational system in the past several years. For example, the average NAEP science score among 12th graders in the US assessed in 2015 is similar to the 2009 score. Sadly, these scores are just above basic and far from proficient or advanced levels [11].

\section{Conclusion}

It is estimated that the US will reach over 500,000 fatal cases by April 2021 [12]. This number could change depending on how we are able to control the spread of the infection at the community level. Primary prevention is key. As health care providers we need to take part in addressing the "other pandemic" and educate our communities especially during this time where we see a second and larger uptake of COVID-19 cases. There is evidence that wearing a mask provides protection against infection spread in the community [13]. However, there is still significant work to be done in our society, despite the evidence and common-sense support; some people still refuse to wear a face mask or adhere to public health recommendations. If we continue along the path of ignorance and denialism it will be challenging to achieve a $70 \%-80 \%$ vaccination rate across 
our communities. In the meantime, fighting ignorance and denialism could have a considerable positive impact if addressed effectively.

\section{Conflicts of Interest}

The author declares no conflicts of interest regarding the publication of this paper.

\section{References}

[1] Jester, B., Uyeki, M.T., Jernigan, D. and Tumpey, M.T. (2019) Historical and Clinical Aspects of the 1918 H1N1 Pandemic in the United States. Virology, 527, 32-37. https://doi.org/10.1016/j.virol.2018.10.019

[2] Prather, A.K., Wang, C. and Schooley, T.R. (2020) Reducing Transmission of SARS-CoV-2. Science, 368, 1422-1424. https://doi.org/10.1126/science.abc6197

[3] Sungnak, W., Huang, N., Bécavin, C., Berg, M., Queen, R., Litvinukova, M., et al. (2020) SARS-CoV-2 Entry Factors Are Highly Expressed in Nasal Epithelial Cells Together with Innate Immune Genes. Nature Medicine, 26, 681-687. https://doi.org/10.1038/s41591-020-0868-6

[4] Centers for Disease Control and Prevention (2020) The Deadliest Flu: The Complete Story of the Discovery and Reconstruction of the 1918 Pandemic Virus. https://www.cdc.gov/flu/pandemic-resources/reconstruction-1918-virus.html

[5] Bootsma, M.C. and Ferguson, N.M. (2007) The Effect of Public Health Measures on the 1918 Influenza Pandemic in US Cities. Proceedings of the National Academy of Sciences of the United States of America, 104, 7588-7593.

https://doi.org/10.1073/pnas.0611071104

[6] Wu, Z. and McGoogan, J.M. (2020) Characteristics of and Important Lessons from the Coronavirus Disease 2019 (COVID-19) Outbreak in China: Summary of a Report of 72314 Cases from the Chinese Center for Disease Control and Prevention. JAMA, 323, 1239-1242. https://doi.org/10.1001/jama.2020.2648

[7] Marshal, M. (2020) COVID-19's Lasting Misery. Nature, 585, 339-341. https://doi.org/10.1038/d41586-020-02598-6

[8] Rubin, R. (2020) As Their Numbers Grow, COVID-19 “Long Haulers” Stump Experts. JAMA, 324, 1381-1383. https://doi.org/10.1001/jama.2020.17709

[9] Diethelm, P. and McKee, M. (2009) Denialism: What Is It and How Should Scientists Respond? European Journal of Public Health, 19, 2-4. https://doi.org/10.1093/eurpub/ckn139

[10] Miller, L.B. (2020) Science Denial and COVID Conspiracy Theories: Potential Neurological Mechanisms and Possible Responses. JAMA, 324, 2255-2256. https://doi.org/10.1001/jama.2020.21332

[11] The Nation's Report Card (2015) Average Science Score for Twelfth-Graders Not Significantly Different Compared to 2009. A 2015 Science Assessment. https://www.nationsreportcard.gov/science_2015/\#scores?grade $=12$

[12] Projection of Deaths by COVID-19 in the United States of America (2020). https://covid19.healthdata.org/united-states-of-america?view=total-deaths\&tab=tre nd

[13] Chu, K.D., Akl, A.E., Duda, S., Solo, K., Yaacoub, S., Schünemann, J.H., et al. (2020) Physical Distancing, Face Masks, and Eye Protection to Prevent Person-to-Person Transmission of SARS-CoV-2 and COVID-19: A Systematic Review and Meta-Analysis. Lancet, 395, 1973-1987. https://doi.org/10.1016/j.jvs.2020.07.040 